\title{
In-home evaluation of efficacy and titration of a mandibular advancement device for obstructive sleep apnea
}

\author{
Daniel J. Levendowski • Todd D. Morgan • \\ John E. Patrickus • Philip R. Westbrook • Chris Berka • \\ Timothy Zavora $\cdot$ Djordje Popovic
}

Published online: 18 January 2007

(C) Springer-Verlag 2007

\begin{abstract}
There is increasing evidence that mandibular advancement devices (MADs) can be an effective treatment for some patients with obstructive sleep apnea, a highly prevalent chronic disease. In this study, the objectives were to objectively assess the effectiveness of MAD therapy using a limited channel recorder, and to develop a model for identifying patients who may be appropriate for MAD therapy as the initial treatment option. Thirty patients were prospectively recruited and studied at two independent dentist offices and the participants' homes. Subjects wore the ARES Unicorder for two nights before insertion of the MAD, and again when the dentist determined that the patient had reached the titration endpoint. Self-reported measures of depression, sleepiness, and quality of life were obtained pre- and posttreatment. The reviewer was blinded to the study status while the physiological signals were being visually inspected. Significant reductions in the
\end{abstract}

D. J. Levendowski $(\bowtie) \cdot$ P. R. Westbrook $\cdot$ C. Berka $\cdot$ T. Zavora Advanced Brain Monitoring, Inc., 2850 Pio Pico Drive, Suite A,

Carlsbad, CA 92008, USA

e-mail: Dan@b-alert.com

T. D. Morgan

Scripps Memorial Hospital,

320 Santa Fe Drive, Suite 105,

Encinitas, CA 72024, USA

\section{J. E. Patrickus}

Northeastern WI Snoring and SA Dental,

2343 E. Mason Street,

Green Bay, WI 54302, USA

D. Popovic

Alfred E. Mann Institute for Biomedical Engineering,

University of Southern California,

Los Angeles, CA 90089, USA apnea/hypopnea index (AHI), hypoxemia measures, and snoring level were observed posttreatment. Twenty-seven of the $30(90 \%)$ patients had a posttreatment AHI (using a $4 \%$ desaturation for hypopneas) below a clinical cut-off of 10. All but one patient (97\%) exhibited at least a $50 \%$ decrease in AHI or had a posttreatment $\mathrm{AHI} \leq 10$. Significant differences in body mass index, weight, and neck circumference in patients with posttreatment AHIs above and below a clinical cut-off of five were identified. The linear regression used to predict the posttreatment AHI using pretreatment data resulted in an $R^{2}$ of 0.68 . The model correctly predicted two patients who were unable to obtain a posttreatment AHI of 10 or less. This study was designed to demonstrate two models of collaboration between a dental sleep medicine specialist and a sleep medicine physician in the monitoring of a patient treated with a MAD. The outcome data suggest that the limited channel recording system can be used as an alternative to laboratory polysomnography to reduce the cost of MAD treatment, and to improve the quality and consistency of posttreatment patient care.

Keywords Sleep apnea $\cdot$ Sleep disordered breathing $\cdot$ Home monitoring $\cdot$ Mandibular advancement device $\cdot$ Outcome assessment

\section{Introduction}

Obstructive sleep apnea (OSA) is the most common disorder observed in the practice of sleep medicine and is responsible for more mortality and morbidity than any other sleep disorder [1]. Although characterized over 40 years ago [2, 3], OSA has only recently gained recognition as one of the world's most prevalent, underdiagnosed disorders $[4,5]$. Due to 
associated morbidity, OSA has been identified as a major public health concern [6]. The disorder is characterized by frequent loud snoring and recurrent failures to breathe adequately during sleep (termed apneas or hypopneas), usually as a result of collapse of the upper airway.

Mandibular advancement devices (MADs) are being increasingly recognized as a treatment alternative to continuous positive airway pressure (CPAP), particularly for patients with mild to moderate OSA [7-12]. MADs are designed to protrude the mandible and thus the tongue and epiglottis during sleep, preventing airway occlusion [13]. Comparisons of MAD to CPAP have revealed that although MADs are less efficacious than CPAP in reducing the RDI they are, on average, used more frequently, preferred by more patients and more readily accepted than CPAP [14]. Recent studies demonstrated that both MADs and CPAP can reduce the 24-h diastolic blood pressure by an average of $3 \mathrm{mmHg}$ after 4 weeks of treatment [15-17]. Similarly to CPAP, self-reported improvements in quality of life and decrease in sleepiness are reported in the majority of patients [14].

The primary goal of this study was to use a self-applied limited channel recorder to objectively assess the effectiveness of MAD therapy when the dentist determined the patient had reached the titration endpoint. A second goal was to determine factors which might predict successful treatment outcomes and provide a more refined method for identifying patients who may be appropriate for MAD therapy as the initial treatment option. Finally, we wanted to demonstrate two potential models of collaboration between the dentist and sleep medicine physician in monitoring MAD treatment outcomes.

\section{Materials and methods}

Eleven females and 19 males were recruited from two dental practices before treatment with an oral appliance and enrolled in the study. Twenty-five of the patients had failed CPAP therapy. After obtaining an informed consent (approved by the BioMed IRB, San Diego, CA, USA) patients completed a two-night pretreatment in-home study with the Apnea Risk Evaluation System (ARES TM) Unicorder TM (Advanced Brain Monitoring, Carlsbad, CA, USA). This pretreatment recording was conducted between the time the dental impressions were taken and when the TAP II Mandibular Advancement Device (MAD) (Airway Management, Dallas, TX, USA) was fabricated and ready for insertion.

The TAP is a custom-made oral appliance with separate upper and lower appliances joined by a titration or advancement mechanism on the upper and a transverse bar or socket on the lower. The titration mechanism uses a hook to engage the bar or socket on the lower once each device is placed in the mouth. A jackscrew controls the position of the hook and thus the amount of protrusion. The patient can self-titrate the device using a removable hex key which engages the screw.

On the day of insertion of the MAD, patients completed the Beck depression index, Epworth sleepiness scale, and the Flemons' quality of life questionnaire. At the insertion appointment, both study sites attempted to achieve a starting MAD titration position whereby the patient could just hook the lower tray with the upper tray using active protrusion with both trays in place. In the rare occasion that this level of advancement was not tolerated by the patient, the starting protrusion was reduced. Patients were instructed to begin adjusting the MAD in one-half turn increments as soon as it was tolerable, until a cessation in snoring or the symptoms had resolved. As a result, the titration endpoint was determined by the dentist based on the patient's selfreport. At the follow-up appointment, which was typically scheduled 3 to 4 weeks subsequent to the MAD insertion, the assessment questionnaires were completed again and the ARES Unicorder study was repeated. Twenty-seven of the 30 patients reached their endpoint within 34 days. The other three patients completed their endpoint in 40,61, and 75 days; the delay in reaching the endpoint was due to patient illness unrelated to this study.

In between the time that treatment was initiated and the titration endpoint, each patient maintained a daily journal that recorded the time the appliance was inserted each night, and the time it was removed in the morning.

The pre- and posttreatment studies were conducted with the ARES Unicorder. From a single site on the forehead, the wireless recorder measures oxygen saturation, pulse rate, airflow, respiratory effort, snoring levels, head movement, and head position [18] (Fig. 1). Reflectance oximetry is used to obtain the $\mathrm{SpO} 2$ and pulse rate signals. Respiratory effort is derived from the measurement of changes in forehead venous pressure acquired using a combination of photoplethysmography and changes in surface pressure of the forehead reflectance oximetry sensor. Airflow is obtained via a cannula and nasal pressure transducer. A calibrated acoustic microphone is used to acquire quantified snoring levels $(\mathrm{dB})$. Actigraphy is used to measure head movement and derive head position. The recorder was designed to be affixed by the patient, and provide alerts during the study if poor quality airflow or $\mathrm{SpO} 2$ is detected so the device could be adjusted.

The description and validation of this device in 284 valid comparisons of the in-laboratory simultaneous PSG and ARES and 187 valid comparisons of the in-laboratory PSG with a separate two nights unattended self-applied ARES Unicorder has been previously published [18]. Using a diagnostic AHI cutoff of $>10$, the concurrent in-lab comparison yielded a sensitivity of 97.4, a specificity of 


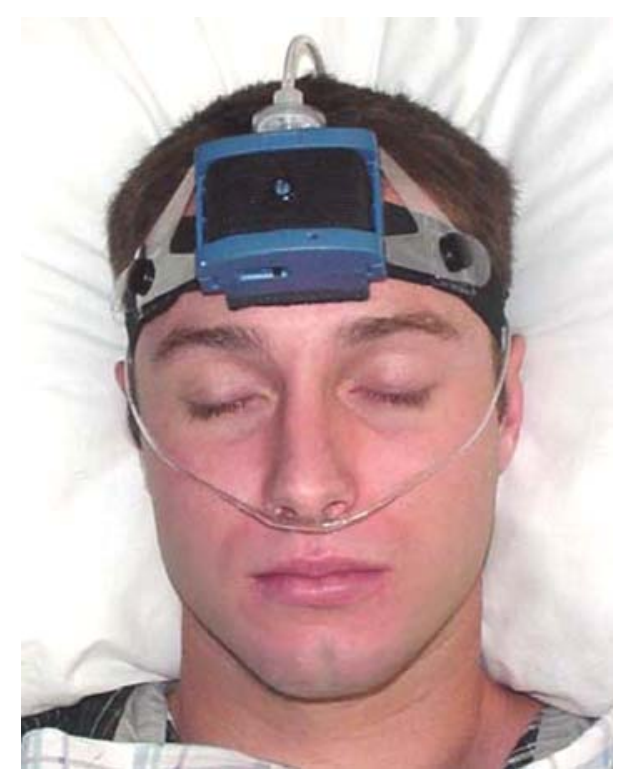

Fig. 1 Patient wearing a unicorder

85.6, a positive predictive value of 93.6 , and a negative predictive value of 93.9. The in-home comparison sensitivity, specificity, positive predictive value, and negative predictive value were $91.5,85.7,91.5$, and 85.7 , respectively. The failure rate was $2 \%$.

In an independent validation of 40 subjects (13 healthy controls and 27 patients with symptoms of EDS and/or snoring) using a clinical cut-off of 15 , the sensitivity of the ARES compared to concurrent PSG was 100\% (25/25 patients) and specificity $67 \%$ (10/15 subjects). The sensitivity for two-nights of ARES in-home compared to the PSG was $96 \%$; specificity was $80 \%$. The failure rate was $5 \%$ [19].

Automated scoring algorithms were applied off-line to detect sleep disordered breathing. The AHI was computed using a time-in-bed measure based on recording time with acceptable signal quality minus periods when the patient was upright or presumed to be awake based on actigraphy. Apneas, based on a 10-s cessation of airflow detected by the automated algorithms, were included in all apneahypopnea indexes (AHI). Hypopnea events required a 50\% reduction and recovery in airflow and were further stratified based on the depth of desaturation. The AHI- $4 \%$ criteria required a minimum $3.5 \%$ reduction in $\mathrm{SpO} 2$ and at least a $1.0 \%$ recovery. Hypopneas included in the AHI- $3 \%$ and AHI-1\% criteria required $\mathrm{SpO} 2$ desaturation and resaturation using stepped thresholds. For the AHI-3\%, if the $\mathrm{SpO} 2$ at the point of maximum saturation before the event was greater than or equal to $95 \%$ then a $2.2 \%$ reduction and a $2.2 \%$ recovery in $\mathrm{SpO} 2$ was required. For maximum saturations of between $95-93 \%$ the required $\mathrm{SpO} 2$ change was a $2.5 \%$ reduction and $2.5 \%$ recovery; between $93-91 \%$ a $3.0 \%$ reduction and $2.7 \%$ recovery; between $91-88 \%$ a $3.5 \%$ reduction and $3.0 \%$ recovery; and below $88 \%$ a $4.0 \%$ reduction and $3.5 \%$ recovery. For the AHI- $1 \%$, if the point of maximum saturation before the event was greater than $93 \%$, then a $1.0 \%$ reduction and $1.0 \%$ recovery was required; and for events with a starting $\mathrm{SpO} 2$ between $93-91 \%$, a $1.2 \%$ reduction and $1.2 \%$ recovery was required. For an AHI- $1 \%$ event to be called, the change in flow and desaturation needed to be associated with a behavioral arousal (i.e., an abrupt change in pulse rate, snoring sound or a head movement). After the automated scoring was applied, the full disclosure recordings were visually inspected by a sleep medicine physician to confirm the accuracy of the automated scoring, and to reclassify as central and/or exclude autodetected events if necessary. At the time of the review, the clinician was blinded to the study status (i.e., pre- or posttreatment). The physiological data, including AHI values, percent time with $\mathrm{SpO} 2$ below 90,85 , and $80 \%$, and percentage time snoring greater than $30,40,50$, and $60 \mathrm{~dB}$ were then calculated.

Responses to the Beck depression index and Flemons' quality of life questionnaire were tallied in accordance with published methods [20, 21].

Paired $t$ tests were used to identify significant changes in the pre- and posttreatment physiological data and questionnaire responses.

To identify anthropomorphic factors that may impact MAD treatment outcomes, patients were stratified into two groups. Group 1 included all patients with a posttreatment AHI- $4 \% \leq 5(n=18)$. The balance of patients with an AHI- $4 \%>5$ were assigned to group $2(n=12)$. Paired $t$ tests were used to identify significant group differences.

To develop and validate the prediction of the posttreatment AHI using pretreatment data, patients were paired and assigned into either the model development or cross validation group based on similarities in the pre- and post- $4 \% \mathrm{AHI}$ and $1 \%$ AHI. Correlation analysis was used to identify anthropomorphic variables and measures of obstructive breathing before treatment which might be useful in estimating the posttreatment $4 \%$ AHI (post-T 4\%). Variables with significant correlations were then used in a linear regression to derive predicted posttreatment values (predict AHI).

\section{Results}

Overall effects of MAD treatment The mean $\pm \mathrm{SD}$ and minimum pre- and posttreatment valid recording times were $9.6 \pm 3.6$ and $3.5 \mathrm{~h}$; and $10.3 \pm 2.4$ and $4.6 \mathrm{~h}$, respectively.

Paired $t$ tests revealed significant changes $(p<0.001)$ in all pre- vs posttreatment sleep disordered breathing measures, including: apnea-hypopnea index with a 4\% (AHI-4\%) and 1\% (AHI-1\%) desaturation (Fig. 2).

A decrease in hypoxemia measures pre- and posttreatment were also observed including the percentage of time 
below 90\% SpO2 $(\mathrm{T} \%<90 \%)(p<0.05)$ and the mean percentage change in $\mathrm{SpO} 2$ across all AHI events (\% Dip) $(p<0.001)$ (see Fig. 3).

Significant reduction in the average snoring levels at 30 and $40 \mathrm{~dB}$ across the valid recording time were also observed ( $p<0.001)$ (Fig. 4).

Based on conventional outcome measures, the MAD therapy was highly efficacious. Twenty-seven of the 30 $(90 \%)$ patients had a posttreatment AHI-4\%<10 (Fig. 5a). All but one patient (97\%) exhibited at least a 50\% decrease in AHI- $4 \%$ or had a posttreatment AHI- $\% \leq 10$.

Using an alternative measure of AHI-1\% (used to identify residual sleep disordered breathing/upper airway resistance) 21 of the 30 patients $(70 \%)$ had a posttreatment AHI- $1 \% \leq 15$ (Fig. $5 b$ ). Eighty-seven percent ( 26 of 30 ) of the patients exhibited at least a $50 \%$ decrease in AHI- $1 \%$ or had a posttreatment AHI- $1 \% \leq 15$.

Paired $t$ tests applied to the pre- and posttreatment scores revealed statistically significant differences for Beck depression index, Flemons' QOL, Epworth sleepiness score (all at the $p \leq 0.001$ level) (Fig. 6). Seventy-six percent (23/ 30 ) of the patients showed a decrease in Epworth score; $60 \%$ had an Epworth score reduction of three or more. Seventy-three percent $(22 / 30)$ of the patients reported a posttreatment reduction in depression; 53\% had a Beck depression index score reduction of three or more. Eightyseven percent of the patients reported an increase in the Flemons' QOL index; 60\% showed in improvement score of one or greater. All patients reported some level of subjective improvement on at least one of these subjective measures.

The correlation between the posttreatment percentage time snoring above $30 \mathrm{~dB}$ and the AHI- $4 \%$ was computed because snoring is a principal measure used to assess the MAD titration endpoint. The results in Fig. 7 suggest that snoring is a good predictor of outcome when objectively measured.

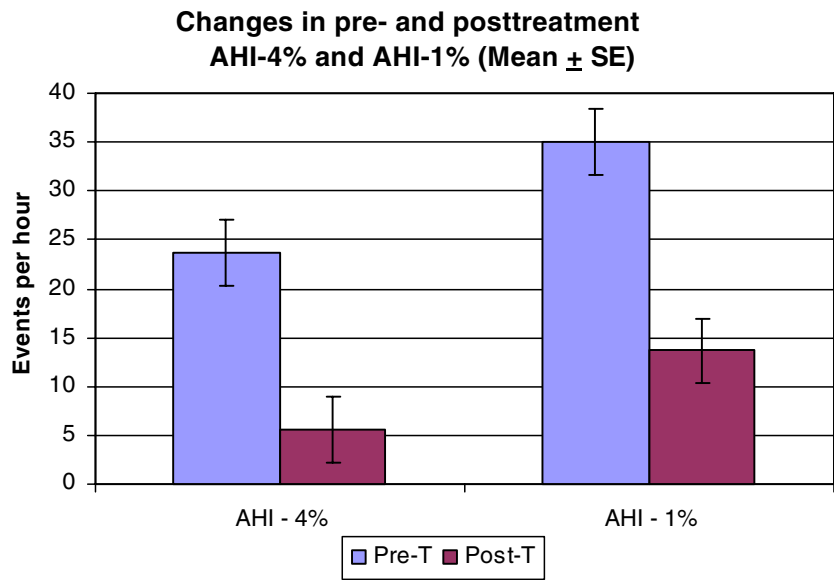

Fig. 2 Mean + SE changes in pre- and posttreatment AHI- $4 \%$ and AHI- $1 \%$

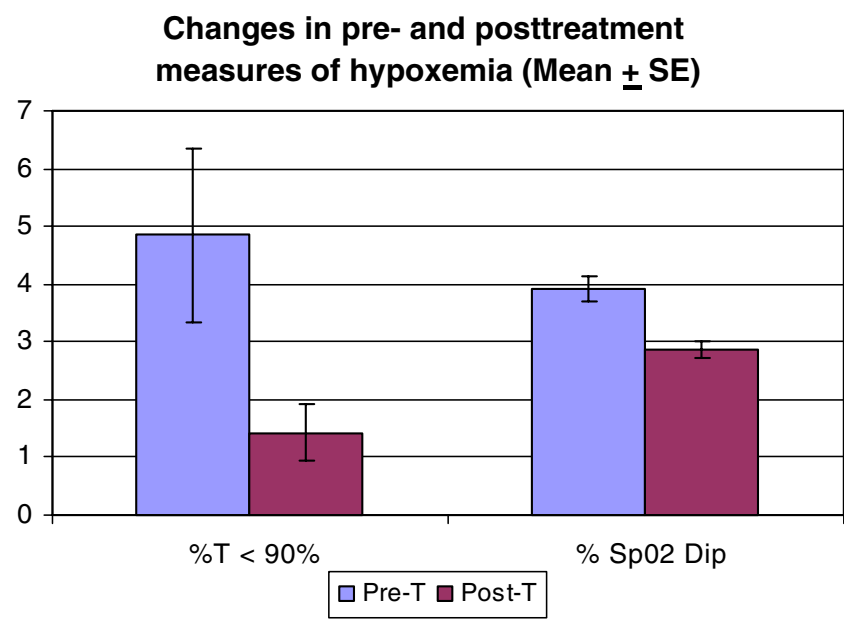

Fig. 3 Mean + SE changes in pre- and posttreatment percentage of time the $\mathrm{SpO} 2$ was $<90 \%$, and the percentage change in $\mathrm{SpO} 2$ resulting from sleep disordered breathing

Daily self-reported compliance for the MAD revealed that out of a total of 693 nights in bed recorded across all subjects, only 20 nights were reported as not wearing the MAD. The mean usage per night was $7.34 \pm 1.3 \mathrm{~h}$. Three of the 30 patients had incomplete paperwork due to either confusion on the part of the patient or problems in collecting the paperwork at the study sites. Although all three patients said that they had worn the MAD for the duration of the study, there was no written record available that allowed the investigators to assess compliance/usage.

Factors that affect treatment outcomes Analysis of variance was used to compare demographic and anthropomorphic measures of patients who had a posttreatment AHI- $4 \% \leq 5$ and those who did not respond optimally to treatment (Table 1). Significant differences in the two

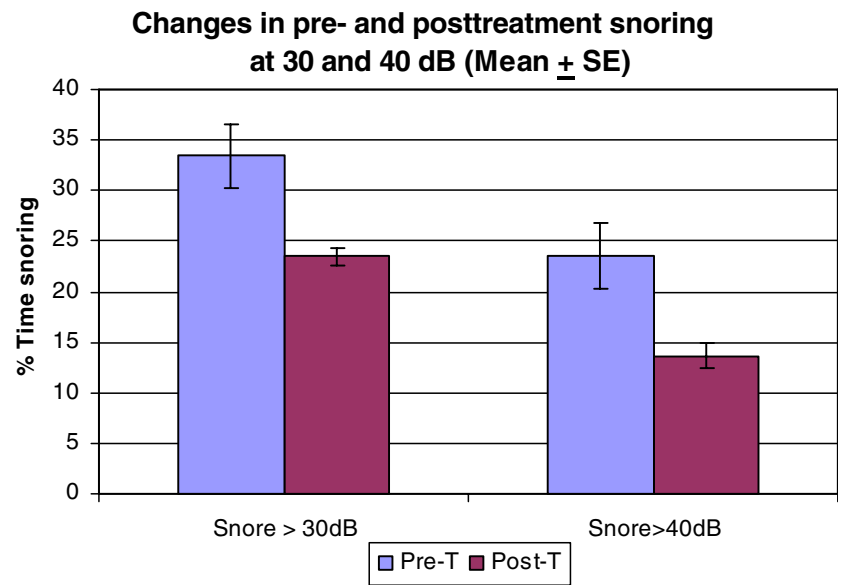

Fig. 4 Mean + SE changes in pre- and posttreatment snoring loudness above 30 and $40 \mathrm{~dB}$ 
a Individual changes in pre- and posttreatment $\mathrm{AHI}-4 \%$

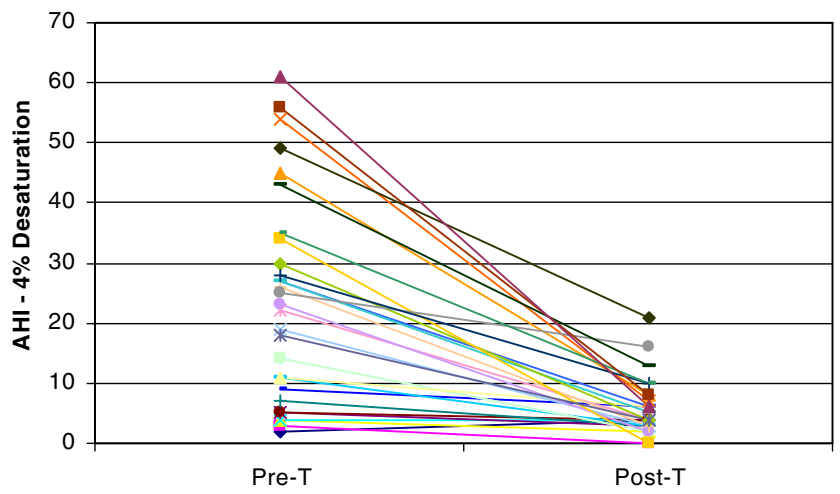

b Individual changes in pre- and posttreatment

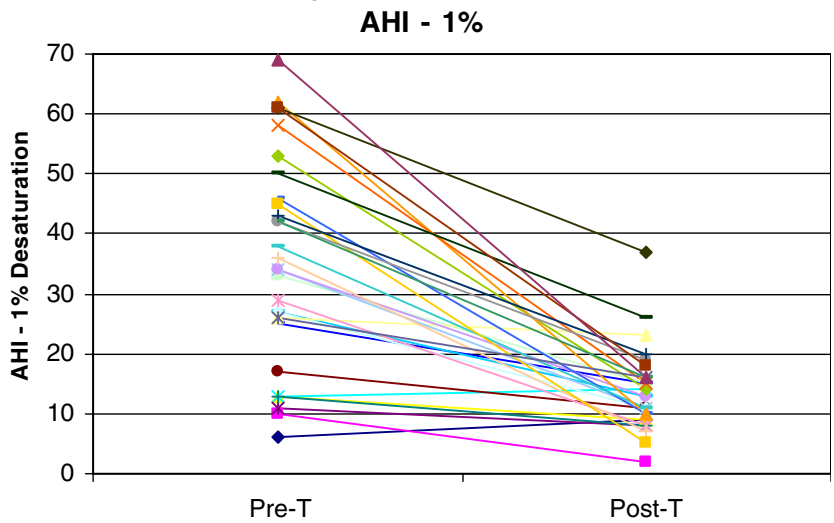

Fig. 5 Individual changes in pre- and posttreatment sleep disordered breathing using: a AHI-4\% desaturation and b AHI-1\% desaturation

groups were observed in the body mass index $(p<0.01)$, weight $(p<0.01)$, and neck circumference $(p<0.02)$.

Prediction of successful treatment outcome Correlation analysis was applied to the entire data set $(n=30)$ identify

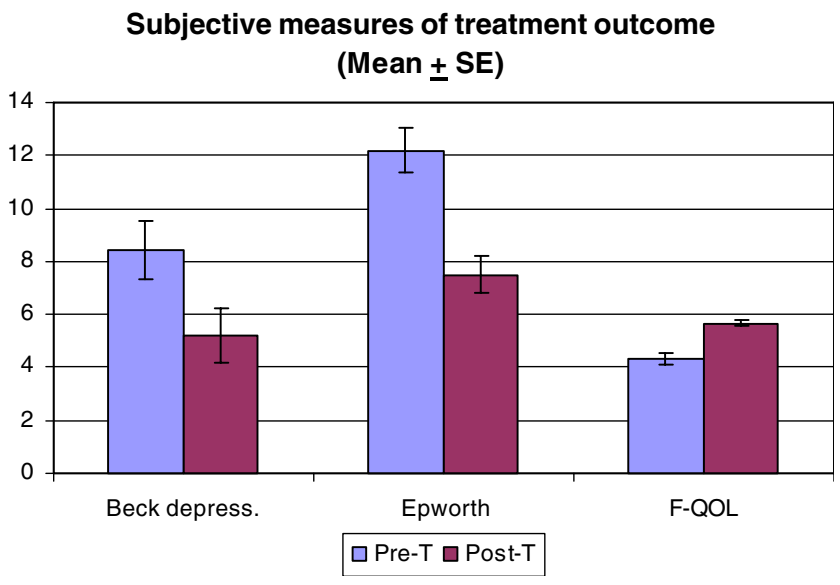

Fig. 6 Pre- and posttreatment changes in Beck depression index, Epworth sleepiness scale, and Flemons' quality of life index
Relationship between posttreatment snoring $>30 \mathrm{~dB}$ and $4 \% \mathrm{AHI}$

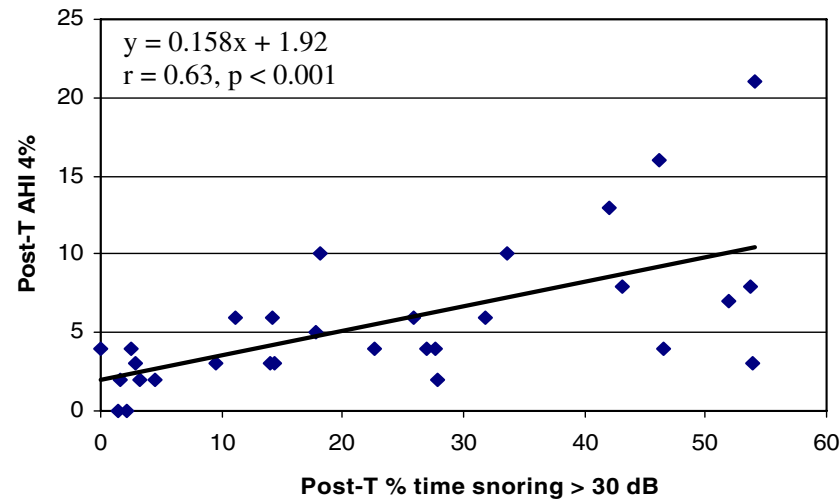

Fig. 7 Regression plot predicting posttreatment AHI-4\% based on the posttreatment percentage of time the snoring loudness was above $30 \mathrm{~dB}$

pretreatment variables that had a significant relationship with the posttreatment AHI-4\% (see Table 2).

Various combinations of variables were submitted to linear regression using the model development data set in an effort to predict the posttreatment AHI-4\%. Limiting the number of variables to four (due to the small sample size), the variables $\mathrm{AHI}-4 \%$, AHI-3\%, AHI- $\%$, and percent time snoring $>30 \mathrm{~dB}$ resulted in an $R^{2}$ of 0.68 . The coefficients derived from the linear regression were applied to the model development and cross-validation data sets.

Twenty-seven of the 30 patients had predict-AHI suggesting a successful outcome would be attained using a clinical cutoff post-T $4 \% \mathrm{AHI} \leq 10$ (Fig. 8). Two of the three patients were accurately predicted as unable to achieve a post- $\mathrm{T} A H I \leq 10$. The Bland-Altman plots comparing the predicted-AHI and posttreatment AHI (Fig. 9) suggest a reasonable fit of the data given the small sample size used for the model development and cross validation data sets.

Table 1 Characteristics of group that was treated optimally and the group that was not treated optionally with a MAD (mean $+\mathrm{SE})$

Treated optimally Did not treat optimally post-T AHI- $4 \% \leq 5$ post-T AHI- $4 \%>5$

Gender

Females

Males

Age (years)

BMI $(\mathrm{kg} / \mathrm{m})^{* *}$

Weight $(\mathrm{kg})^{* *}$

Neck

7

11

4

$48.3 \pm 2.5$

$50.9 \pm 3.4$

$27.6 \pm 0.8 \quad 33.1 \pm 2.1$

$84.4 \pm 3.0 \quad 100.2 \pm 5.0$

$40.0 \pm 0.7 \quad 43.2 \pm 1.0$

\section{$* p<0.05$}

$* * p<0.01$ 
Table 2 Correlations between posttreatment $\mathrm{AHI}-4 \%$ and pretreatment measures

\begin{tabular}{llll}
\hline Pearson $r(p<0.01)$ & & Pearson $r(p<0.05)$ & \\
\hline Pretreatment 4\% AHI & $r=0.53$ & Snoring $>30 \mathrm{~dB}$ & $r=0.44$ \\
Pretreatment 3\% AHI & $r=0.44$ & Neck circumference & $r=0.45$ \\
Pretreatment $1 \%$ AHI & $r=0.54$ & Body mass index & $r=0.45$ \\
Snoring $>40 \mathrm{~dB}$ & $r=0.52$ & Weight & $r=0.45$ \\
\% Time $<90 \%$ SpO2 & $r=0.56$ & & \\
\hline
\end{tabular}

\section{Discussion}

Consecutive patients who were referred to the dentist for a MAD were provided the opportunity to enroll in the study. The only exclusion criteria applied were ages less than 18 or older than 70 years. The inclusion of 25 subjects who had previously failed CPAP therapy contributed to the wide OSA severity range: nine of the subjects $(30 \%)$ were considered to have severe OSA (i.e., AHI- $4 \% \geq 30$ ) and an additional $30 \%$ were considered to have moderate OSA (AHI-4\%>15 and <30). Seventy-seven percent of the patients $(23 / 30)$ had at AHI- $1 \%>25$, a clinical cut-off which was considered at least moderate.
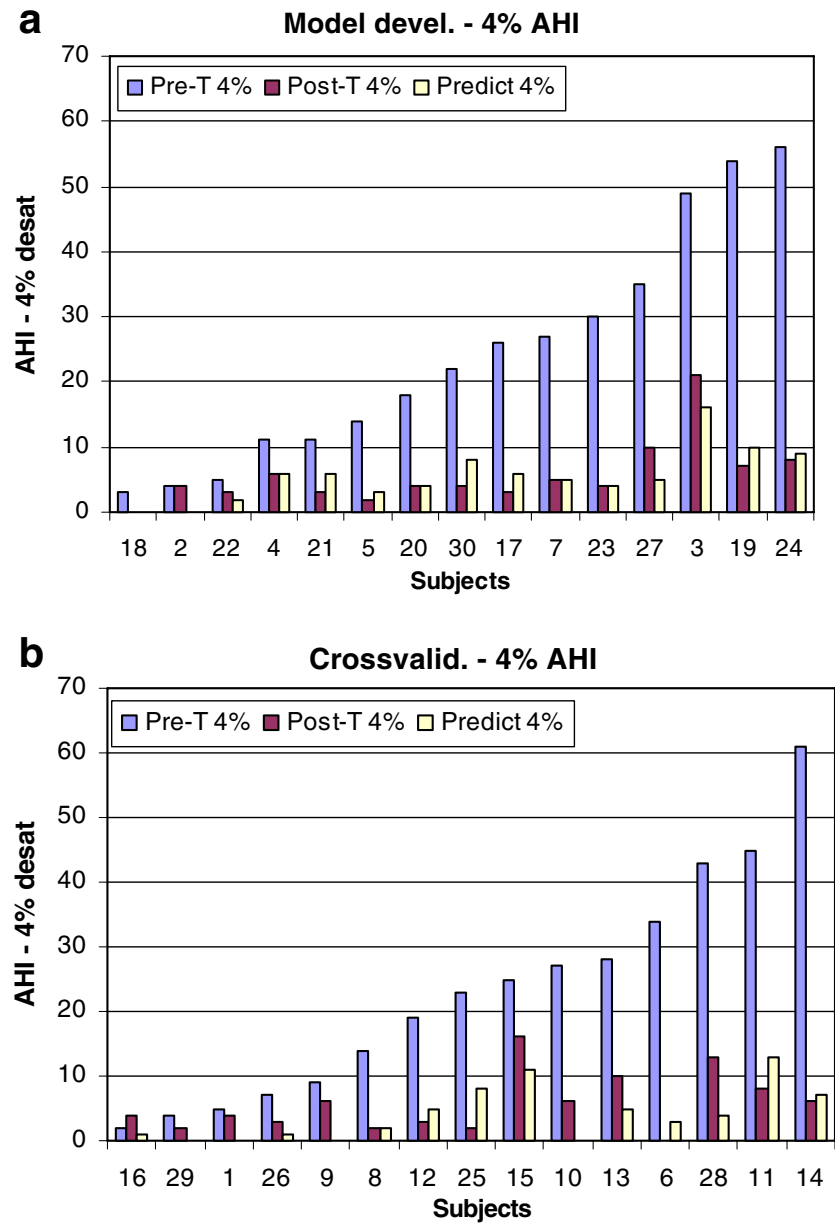

Fig. 8 Comparison of pretreatment, posttreatment, and predicted posttreatment AHI- $4 \%$ for a the model development, and $\mathbf{b}$ the cross validation groups
In this study, a treatment efficacy rate for the MAD was $90 \%$ using an AHI- $4 \%$ clinical cut-off of 10 , and $97 \%$ when a $50 \%$ reduction in AHI was included in determining a successful outcome. Eighty-seven percent of the patients showed improvement using an AHI-1\% with a clinical cutoff of 15 or a $50 \%$ reduction in AHI. Eighty percent of the patients $(24 / 30)$ had a posttreatment AHI- $1 \% \leq 16$.

A recent evidence-based review summarized results from 87 published studies (over 2,000 patients evaluated) including 15 Sackett Level I and Level II randomized controlled trials [14]. Using a definition of treatment
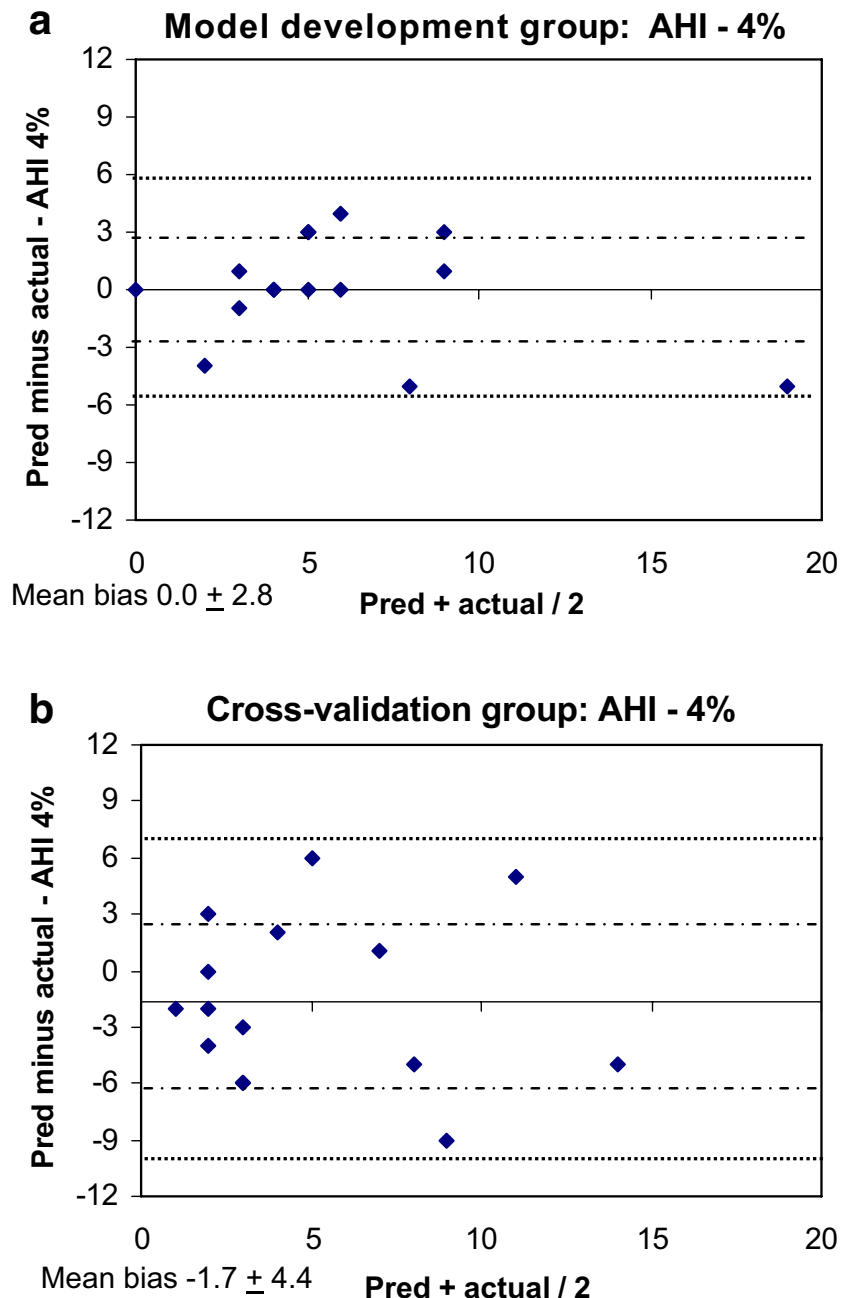

Fig. 9 Bland-Altman plot of the difference between the predicted posttreatment $\mathrm{AHI}-4 \%$ vs the actual posttreatment AHI for a the model development and $\mathbf{b}$ the cross validation groups 
success that required achieving a posttreatment respiratory disturbance index below 10, success was achieved on average across all studies for $52 \%$ of patients treated with oral appliances. When the more liberal criteria of a $50 \%$ reduction in RDI were applied to assess outcome, $65 \%$ of patients were shown to have a positive result with MAD.

In an attempt to compare these previous finding to this data set, a clinical cut-off of 10 was applied to the AHI- $1 \%$. The differences between the AHI- $1 \%$ and RDI include a $50 \%$ reduction in flow vs $30 \%$; a $1 \%$ desaturation vs no desaturation; arousal indicators based on changes in snoring, pulse rate, and head movement vs cortical arousals; and the use of time-in-bed vs total-sleep-time. Using this alternative clinical cut-off, $33 \%$ of the patients had a successful outcome. When a $50 \%$ reduction in AHI$1 \%$ was included, $77 \%$ had a successful outcome. Results are more consistent with the previous research.

The findings in this study were consistent with previous reports which found self-reported improvements in quality of life and decrease in sleepiness in the majority of patients [14]. No effort was made in this study to obtain objective measures of improvement. To date, only four studies have utilized objective measures of alertness or neurocognitive function to assess treatment outcomes with MAD therapy, with mixed results [22-25]. Further investigations need to be conducted to assess the effectiveness of MAD therapy in ameliorating cognitive impairments in OSA.

The strong correlation $(r=0.63)$ between posttreatment $\%$ time snoring $>30 \mathrm{~dB}$ and the posttreatment AHI- $4 \%$ suggests that the current clinical practice of relying on residual snoring to asses MAD titration is valid. It is interesting to note that there was no apparent correlation between snoring levels and pretreatment AHI values. Given the discrepancy between the self-reported and objectively measured snoring at the titration endpoint, it appears that the bed-partner or patient's observation may not provide the most accurate means for titrating a MAD.

Several studies have reported increased efficacy for MADs in patients with supine positional OSA [7, 26]; however, the association has not been widely confirmed. This is due in part to the fact that the majority of studies on MAD therapy do not report the number of abnormal respiratory events by sleep position despite the recommendation of the ASDA Practice Parameters for separate calculations of supine and lateral RDIs [27, 28]. Supine positionality did not appear to influence the treatment outcome in our study, possibly, because there were so few MAD treatment failures. This may be a result of the appliance which was used or that the small sample size was not representative of the population treated with oral appliances.

A recent evidence-based review reported that median MAD treatment compliance across patients was $77 \%$ after 1 year [14]. In this study, compliance was only monitored during the titration period which may have contributed to the favorable finding (i.e., 97\%). Other factors that may have influenced this outcome were a relatively small sample size and/or patient compensation being provided. Given that $80 \%$ of the study participants were CPAP failures, the influence of prior CPAP use on MAD compliance should be explored. While an objective measure of MAD compliance would eliminate potential bias contributed by self-reported use, practical methods are not currently available.

The suggested decrease in MAD efficacy with increasing body mass index (BMI) was confirmed [27, 28]. Weight and neck circumference also appeared to influence the posttreatment apnea/hypopnea index. These variables make sense: the upper airway tends to be narrower in patients with more fatty tissue around the neck and the additional mass combines with gravity to contribute to greater collapsibility when sleeping supine.

The successful treatment outcome of patients with severe sleep apnea suggests that a more quantitative approach should be investigated to identify candidates appropriate for a MAD therapy. The results from the predictive model, once fully validated, could provide the guidance needed for sleep medicine physicians to recommend an oral appliance as an initial treatment option for more severe patients. Alternatively, substantial differences between the predicted and actual posttreatment AHI could help dentists determine when a patient has not been fully titrated.

Given the small sample size of the model development data set $(n=15)$, only four variables were included in the regression model even though the correlations presented in Table 2 suggested additional variables would be predictive. It is expected that the error between the predicted and actual posttreatment AHI can be reduced with larger data sets. In addition to expanding the database used for the predictive model, future investigations should be conducted to determine if the accuracy of the predictive model is influenced by the type of MAD.

Dentists represent an important access point for identifying and treating patients with undiagnosed OSA. This study was designed to demonstrate two models of collaboration between a dental sleep medicine specialist and a sleep medicine physician. As recommended by the AASM, only patients with mild to moderate OSA were offered MAD therapy as the initial treatment choice in this study. Patients with severe sleep apnea were enrolled only after failure of CPAP therapy. In one model, the dentist referred the patient to the sleep medicine physician and his staff to obtain and review the pre- and posttreatment physiological data. In the second model, the dentist acquired the data and transmitted it to the sleep medicine physician for review. In both models, the physical and history was made available to the physician for interpretation of the data [29]. 
A follow-up PSG is generally not affordable in cases where it is not covered by an insurance company or health ministry; sleep centers do not tend to offer less expensive level III studies as an alternative. This creates a situation whereby treatment outcomes are simply not assessed, or the dentist conducts the study independently, without the assistance or oversight of the sleep medicine professional. Left on their own, many dentists rely upon oximetry and other level IV devices to monitor treatment outcomes, in part because of their limited experience in interpreting the more sophisticated signals obtained by level III devices. Although several level III devices have automated scoring algorithms, the physiological data should still be reviewed by a trained professional. In two patients, the review of the full disclosure recording allowed recognition of complex sleep apnea (i.e., central sleep apnea was revealed posttreatment after the obstructive breathing was resolved) suggesting the importance of having an experienced professional review the data. This study suggests an alternative approach for assessing MAD treatment outcomes that is based on a collaborative relationship between dentists and sleep medicine physician using a limited channel recording system.

Acknowledgements The authors wish to thank Michelle Lumicao, Melissa Whitmoyer, Suzette Grossman, and Kristy L. Wyckoff for their assistance in coordinating the study. This work was supported by the National Institute of Dentistry and Craniofacial Research (DE016772). Daniel J. Levendowski, Philip R. Westbrook, Chris Berka, and Timothy Zavora are employees and shareholders of Advanced Brain Monitoring. Djordje Popovic was a paid consultant of Advanced Brain Monitoring.

\section{References}

1. US Department of Health and Human Services (2000) Healthy People 2010. US Department of Health and Human Services, Washington, DC

2. Gastaut H, Tassinari CA, Duron B (1965) Polygraphic study of diurnal and nocturnal (hypnic and respiratory) episodal manifestations of Pickwick syndrome. Rev Neurol (Paris) 112(6):568-579

3. Jung R, Kuhlo W (1965) Neurophysiological studies of abnormal night sleep and the pickwickian syndrome. Prog Brain Res 18:140-159

4. Dement WC, Vaughan CC (1999) In: House R (ed) The promise of sleep: a pioneer in sleep medicine explores the vital connection between health, happiness, and a good night's sleep. Delacorte Press, New York

5. Kripke DF, Ancoli-Israel S, Klauber MR, Wingard DL, Mason WJ, Mullaney DJ (1997) Prevalence of sleep-disordered breathing in ages 40-64 years: a population-based survey. Sleep 20(1): 65-76

6. Phillipson EA (1993) Sleep apnea-a major public health problem. N Engl J Med 328(17):1271-1273

7. Marklund M, Persson M, Franklin KA (1998) Treatment success with a mandibular advancement device is related to supinedependent sleep apnea. Chest 114(6):1630-1635
8. Marklund M, Steunlund H, Franklin KA (2004) Mandibular advancement devices in 630 men and women with obstructive sleep apnea and snoring: tolerability and predictors of treatment success. Chest 125:1270-1278

9. Clark GT, Blumenfeld I, Yoffe N, Peled E, Lavie P (1996) A crossover study comparing the efficacy of continuous airway pressure with anterior mandibular positioning devices on patients with obstructive sleep apnea. Chest 109(6):1477-1483

10. Ferguson KA, Ono T, Lowe AA, Al-Majed S, Love LL, Fleetham JA (1997) A short-term controlled trial of an adjustable oral appliance for the treatment of mild to moderate obstructive sleep apnea. Thorax 52(4):362-368

11. Eveloff SE, Rosenberg CL, Carlisle CC, Millman RP (1994) Efficacy of a Herbst mandibular advancement device in obstructive sleep apnea. Am J Respir Crit Care Med 149:905-909

12. Pancer J, Al-Faift S, Al-Faift M, Hoffstein V (1999) Evaluation of variable mandibular advancement appliance for treatment of snoring and sleep apnea. Chest 116(6):1511-1518

13. Ryan CF, Love LL, Peat D, Fleetham JA, Lowe AA (1999) Mandibular advancement oral appliance therapy for obstructive sleep apnoea: effect on awake calibre of the velopharynx. Thorax 54(11):972-977

14. Ferguson KA, Cartwright R, Rogers R, Schmidt-Novara W (2006) Oral appliances for snoring and obstructive sleep apnea: a review. Sleep 29(2):244-262

15. Faccenda JF, Mackay TW, Boon NA, Douglas NJ (2001) Randomized placebo-controlled trial of continuous positive airway pressure on blood pressure in the sleep apnea-hypopnea syndrome. Am J Respir Crit Care Med 163(2):344-348

16. Pepperell JC, Ramdassingh-Dow S, Crosthwaite N, Mullins R, Jenkinson C, Stradling JR, Davies RJ (2002) Ambulatory blood pressure after therapeutic and subtherapeutic nasal continuous positive airway pressure for obstructive sleep apnoea: a randomised parallel trial. Lancet 359(9302):204-210

17. Gotsopoulos H, Kelly J, Cistulli P (2004) Oral appliance therapy reduces blood pressure in obstructive sleep apnea: a randomized controlled trial. Sleep 27(5):934-941

18. Westbrook P, Levendowski D, Cvetinovic M, Zavora T, Velimirovic V, Henninger D, Nicholson D (2005) Description and validation of the apnea risk evaluation system: a novel method to diagnose sleep apnea-hypopnea in the home. Chest 128(4):2166-2175

19. Ayappa I, Rapoport D, Westbrook P, Levendowski D, Zavora T, Norman R (2006) Validation of a self-applied unattended monitor for Sleep Disordered Breathing (SDB). Sleep 29(Abstract Supplement 29):A343

20. Beck A (1978) Beck depression inventory. The Psychological Corporation. Harcourt Brace Jovanovich, San Diego, CA

21. Flemons WW, Reimer MA (1998) Development of a diseasespecific health-related quality of life questionnaire for sleep apnea. Am J Respir Crit Care Med 158(2):494-503

22. Gotsopoulos H, Chen C, Qian J, Cistulli P (2002) Oral appliance therapy improves symptoms in obstructive sleep apnea. Am J Respir Crit Care Med 166:743-748

23. Barnes M, McEvoy RD, Banks S, Tarquinio N, Murray CG, Vowles M, Pierce RJ (2004) Efficacy of positive airway pressure and oral appliance in mild to moderate obstructive sleep apnea. Am J Respir Crit Care Med 170:656-664

24. Engleman H, McDonald JP, Graham D, Lello GE, Kingshott RN, Coleman EL, Mackay TW, Douglas NJ (2002) Randomized crossover trial of two treatments sfor sleep apnea/hypopnea syndrome: continuous positive airway pressure and mandibular repositioning splint. Am J Respir Crit Care Med 166:855-859

25. Menn SJ, Loube DI, Morgan TD, Mitler MM, Berger JS, Erman MK (1996) The mandibular repositioning device: role in the treatment of obstructive sleep apnea. Sleep 19(10):794-800 
26. Yoshida K (2000) Effects of mandibular advancement device for the treatment of sleep apnea syndrome and snoring on respiratory function and sleep quality. J Cranio-mandib Pract 18:98-105

27. Association, A.S.D. (1995) Practice parameters for the treatment of snoring and obstructive sleep apnea with oral appliances. Sleep 18(6):511-513

28. Kushida C, Morgenthaler T, Littner M, Alessi CA, Bailey D, Coleman JA Jr, Friedman L, Hirshkowitz M, Kapen S, Kramer
M, Lee-Chiong T, Owens J, Pancer J (2006) Practice parameters for the treatment of snoring and obstructive sleep apnea with oral appliances: an update for 2005. Sleep 29 (2):240-243

29. American Academy of Sleep Medicine (2006) Portable monitoring in the diagnosis of obstructive sleep apnea, an interim statement from the American Academy of Sleep Medicine. J Clin Sleep Med p333 